

\title{
L'éternel passé de la langue: temps et perception linguistique au XVIIe siècle
}

\author{
Gilles Siouffi
}

\section{To cite this version:}

Gilles Siouffi. L'éternel passé de la langue: temps et perception linguistique au XVIIe siècle. Littératures classiques, 2001, 43, p. 241-255. hal-03124667

\section{HAL Id: hal-03124667 \\ https://hal.science/hal-03124667}

Submitted on 28 Jan 2021

HAL is a multi-disciplinary open access archive for the deposit and dissemination of scientific research documents, whether they are published or not. The documents may come from teaching and research institutions in France or abroad, or from public or private research centers.
L'archive ouverte pluridisciplinaire HAL, est destinée au dépôt et à la diffusion de documents scientifiques de niveau recherche, publiés ou non, émanant des établissements d'enseignement et de recherche français ou étrangers, des laboratoires publics ou privés. 


\section{Gilles Siouffi}

«L'éternel passé de la langue : temps et perception linguistique au XVIIe siècle », Littératures classiques, numéro 43,2001 , p. 241-255.

\section{L'éternel passé de la langue : Temps et perception linguistique au $\mathrm{XVII}^{\circ}$ siècle}

Un étrange paradoxe gouverne la description que le $\mathrm{XVII}^{\circ}$ siècle nous a laissée du français. D'un côté, en effet, celle-ci a commencé par se construire dans l'examen d'un abondant matériau historique, où la physionomie d'un idiome unique ne se lisait pas toujours clairement ; de l'autre, elle s'est achevée sur la proclamation d'un état pour ainsi dire "hors-temps" d'une langue, le français, dont la cohérence, le "génie" et l'immortalité ne faisaient pas de doutes. Ce cheminement idéologique et de représentations mérite un examen tout particulier. En effet, la manière dont le présent d'une langue est abordé vis-à-vis de son passé 
n'est jamais anodine. On peut y voir la manifestation privilégiée d'enjeux politiques et culturels; on peut y voir le reflet d'une attitude générale face au temps, dans ce que celui-ci peut représenter de menace ou de promesse à l'égard d'une civilisation ; on peut y voir enfin l'une des figures de l'ambiguïté majeure qui commande, de principe, toute tentative de description d'une langue.

Notre propos, ici, sera d'interpréter quelques-unes des étapes par lesquelles la conscience linguistique française sera passée pour aboutir à un revirement aussi radical. Le français, pour faire bref, aura indiscutablement acquis, au terme du siècle, le statut d'une "langue", mais il l'aura fait au prix d'une dénégation d'une partie de son histoire. Le sentiment diachronique et le sentiment synchronique, que l'on conçoit volontiers, dans nos termes linguistiques modernes, comme les deux volets d'une représentation centralisée de l'idée de langue, n'agissent pas alors à proprement parler en collaboration : ils s'affrontent plutôt dans une lutte qui surprend encore par l'énergie polémique qu'on y a investi. Partisans de l"'histoire" du français, et partisans de son génie contemporain et éternel se sont combattus tout au long du $\mathrm{XVII}^{\circ}$ siècle avec tous les arguments linguistiques que l'on pouvait imaginer. Clairement, ce sont les seconds qui l'ont emporté. La comparaison des débats au centre desquels la langue française fut mise au début et à la toute fin du XVII ${ }^{\circ}$ siècle est à cet égard révélatrice. Si le début du XVII ${ }^{\circ}$ siècle est le moment où la conscience linguistique s'organise autour d'un 
essai de repérage, dans le paysage européen, de tout ce qu'on pourrait appeler des "langues", la transition des $\mathrm{XVI}^{\circ}$ et $\mathrm{XVII}^{\circ}$ siècles se marque aussi par l'effacement graduel de la profondeur de champ dans la vision. La conscience linguistique préfère prendre appui, plus que sur la cohérence d'un parcours historique, sur un sentiment de stabilité dans l'état actuel de la langue. C'est ainsi que la description linguistique, véritablement, naît. Face à la puissance dissolvante du temps, l'approfondissement de la grammaire, la caractérisation de pratiques lexicales et la définition d'un "bon usage" paraissent des moyens d'assurer une identité.

Pour comprendre à partir de quelle situation complexe, dans l'usage, la conscience linguistique du XVII ${ }^{\circ}$ siècle a raisonné, on peut s'aider des analyses de $D$. Trudeau1. Celle-ci rappelle que pour caractériser l'usage linguistique du $\mathrm{XVI}^{\circ}$ siècle en France, il faut avoir recours à un schéma à trois niveaux. Il faut tout d'abord faire état de l'uniliguisme des gens du peuple (dialectes) ; ensuite de la diglossie fréquente des classes sociales favorisées (dialecte avec les domestiques/ français avec les gens du monde) ; enfin de la triglossie des érudits (dialecte de leur province dans la vie quotidienne/français dans les relations sociales/latin dans l'activité intellectuelle). Ainsi, et non sans paradoxe, la synchronie, l'unilinguisme étaient du côté $\mathrm{du}$ peuple. Du côté des savants, on peut noter qu'était fréquent un sentiment de labilité des langues modernes. Ainsi Montaigne commente-t-il son usage du français dans les Essais : "J'escris mon livre à peu d'hommes et à peu d'années. Si ç'eust esté une matiere de durée, il l'eust fallu commettre à un langage plus ferme. Selon la variation

${ }^{1}$ D. Trudeau, Les inventeurs du bon usage, Paris, Minuit, 1992. 
continuelle qui a suivy le nostre jusques à cette heure, qui peut esperer que sa forme presente soit en usage, d'icy à cinquante ans"2. Ce sentiment fait l'objet d'un ouvrage particulier, celui de Louis Le Roy, intitulé De la Vicissitude ou variete des choses en l'univers (1575). Il y est dit entre autres : "Semblablement le temps faict descheoir les parolles, et l'usage en faict de nouveau recroistre d'autres, et leur donne grace, jusques a ce qu'estant consommees peu a peu par vieillesse, elles viennent aussi à-mourir ; parcequ'a la fin et nous et toutes nos choses sont mortelles" (Louis Le Roy, De la Vicissitude ou variete des choses en l'univers, 1575, in Huchon, 5). Et : "Faute d'auteur digne d'être bien suivi, nous n'avons rien de certain, sinon en tant que l'usage commun le reçoit ou le rejette, qui change tous les jours" (Ibid., AV)

Face aux langues modernes, les langues anciennes ont pour elles l'antiquité". Par "antiquité", on ne comprendra pas seulement une très grande ancienneté de fait, mais aussi le présupposé d'une autorité issue d'une capacité à résister de façon définitive au passage du temps. De cette résistance, les langues anciennes semblent alors avoir le monopole. Pour la conscience linguistique, elles ont été illustrées une bonne fois pour toutes par les poètes dont les noms sont célèbres, et sont pour ainsi dire sorties de l'histoire. C'est ainsi que la référence constante aux langues anciennes aide à construire un schéma de représentations des langues à deux niveaux. Les langues sont susceptibles de connaître une vie dans l'histoire, et une vie immortelle, "antique", une fois qu'elles sont sorties de l'immanence historique. Ce double schéma aide à comprendre que l'idéalisation

${ }^{2}$ Montaigne, Essais, livre III, chapitre 9, Paris, club français du livre, 1962, p. 1065. 
linguistique ne soit pas parallèle, jusqu'à la fin du $\mathrm{XVI}^{\circ}$ siècle, à l'exploitation des langues qui en est faite par l'usage. Aussi l'"illustration" des langues modernes se faitelle principalement, à cette époque, à l'aide de traductions (Dolet, Amyot, Blaise de Vigenère) dont le contrat de lecture est souvent double : faire entendre une langue moderne, certes, mais faire entendre aussi, dessous, une seconde voix, celle de la langue antique traduite, qui va se mêler à la première de façon à la faire sortir de la contingence historique. De même remarque-t-on chez Montaigne comme chez beaucoup d'érudits un usage fréquent de citations latines au sein même de l'écriture en langue moderne. Ce dispositif vise clairement à "étoffer", pour ainsi dire, la voix de la langue moderne, et à éviter que le propos ne subisse la caducité encore presque immanquablement associée à l'usage des vernaculaires.

Si le $\mathrm{XVI}^{\circ}$ siècle est le siècle d'une recherche d'"illustration" des langues modernes, on comprend donc que celle-ci se soit aussi faite dans la recherche d'une "antiquité" des vernaculaires. C'est le sens de la démarche d'Etienne Pasquier dans le livre VIII de ses Recherches de la France. La recherche historique appliquée aux langues modernes se veut d'abord un moyen de leur faire rejoindre la manière d'empyrée an-historique où se trouvent les langues anciennes. Dans ses Recherches, Pasquier s'applique à débusquer dans tous les domaines les racines d'une "antiquité nationale". Pour lui, la langue française est comme formée de strates : "Ainsi, la langue dont nous usons aujourd'hui selon mon jugement est composée, part de l'ancienne Gauloise, part de la Latine, part de la Françoise, et si ainsi le voulez, elle a plusieurs grandes 
symbolisations avec la Grégeoise" 3 . Cette vision hétérogène est encore celle d'un homme du $\mathrm{XVI}^{\circ}$ siècle ${ }^{4}$. A vrai dire, Pasquier se montre prudent dans son évaluation du statut des idiomes modernes. Il préfère s'incliner devant la force $\mathrm{du}$ temps : "les langues se changent par une taisible alluvion", dit-il ${ }^{5}$; ou encore : "Il n'est pas dit que tout ce que nous avons changé de l'ancienneté, soit plus poly, ores qu'il ait aujourd'hui cours"6 ; ou encore : "Chacun se fait accroir que la langue vulgaire de son temps est la plus parfaite, et chacun est en cecy trompé"7.

Pour autant, ses Recherches doivent répondre au désir grandissant de voir établis par l'étude, dans les langues modernes, quelques principes échappant à l'usage. Ici, sa réponse sera encore celle d'un homme du $\mathrm{XVI}^{\circ}$ siècle : le statut des langues, leur état possiblement plus élevé (celui-là même qui fonde l'"antiquité" des langues anciennes), ce sera toujours d'abord la puissance supérieure des auteurs qui les utilisent, et de leurs productions. "Quoy doncques ? Dirons nous que les langages ressemblant aux rivieres, lesquelles demeurans tousjours en essence, toutefois il y a un continuel changement des ondes : aussi nos langues vulgaires demeurans en leur general, il y ait changement continu de paroles particulieres, qui ne reviennent plus en usage ? Je vous diray ce que j'en pense. Je croy que l'abondance des bons Autheurs, qui se trouvent en un siecle,

${ }^{3}$ E. Pasquier, Recherches de la France, Paris, Champion, 1996, p. 1498.

${ }^{4} \mathrm{cf}$. Claude Fauchet, Recueil de l'origine de la langue et poésie françoise, ryme et romans, Paris, Patisson, 1581 (Reprints Slatkine, 1972).

5 op. cit., p. 1496.

6Ibid., p. 1519.

7Ibid., p. 1525. 
authorise la langue de leur temps par dessus les autres : On a recours à leurs conceptions originaires, qu'il faut puiser d'eux"8.

Ainsi, pour Pasquier, la conscience linguistique serat-elle avant tout conscience de la continuité d'un passé littéraire et langagier. Celle-ci se révèle dans les préoccupations touchant l'origine de la langue et ce qu'en révèlent les étymologies. D'un autre côté, toutefois, la sensibilité de Pasquier au passage du temps lui fait prendre en compte les problèmes véritablement actuels qui se posent aux langues modernes : les problèmes touchant l'orthographe, par exemple, tels que les soulèvent les propositions de réforme, ou encore ceux afférant, plus généralement, à l'usage. La conscience que Pasquier a du français apparaît ainsi véritablement partagée en deux, saisie entre une tentative d'application au français de la recherche d'autorité que suppose la théorie de l'"antiquité" des langues, et le sentiment de leur hétérogénéité présente.

$\mathrm{Au} \mathrm{XVII}^{\circ}$ siècle, plusieurs auteurs suivront cette piste. Pierre Borel, par exemple, dans son Trésor de Recherches et Antiquitez Gauloises et françoises (1655). Toutefois, de plus en plus, le temps apparaîtra comme un facteur de corruption des langues, et non plus comme un continuel et somme toute naturel "changement d'ondes". En cette première moitié du $\mathrm{XVII}^{\circ}$ siècle, il est clair que les travaux menés sur le français s'efforcent de construire face à l'usage les principes d'une sécurité linguistique. Celle-ci apparaît d'autant plus nécessaire que le français paraît à tous connaître une évolution si radicale qu'elle semble faire passer la langue d'un état "ancien" à un état "moderne". Pour Pasquier, la langue de Marot, par exemple, était une

8Ibid., p. 1526. 
"bigarrure du langage vieux et nouveau" (Huchon, 19). De même, Nicot initule son "trésor" de 1606, le premier grand dictionnaire du français, trésor de la langue française "tant ancienne que moderne", sous le principe, sans doute, que des recherches conjointes sur ces deux états de langue pourrait se déduire un effet cumulatif.

Le dernier représentant peut-être, et le plus notable, de ce courant de recherches historiques, est Ménage, dont les Origines de la langue française paraissent à Paris en 1650. A l'intérieur de la recherche historique, Ménage s'est attaché à occuper un créneau technique et exclusif, celui de l'étymologie. On pourra remarquer que, dans son principe même, la recherche étymologique dénote une sensibilité à la langue et au temps d'un type particulier. Subtilement, en effet, elle suppose que l'investigation du passé va permettre de mettre à jour un état ancien des mots radicalement différent de leur état actuel, immobilisant ainsi celui-ci sous le jour de son "étymologie". C'est ainsi que la recherche étymologique est bien souvent, paradoxalement, une recherche conservatrice de l'état actuel des mots. On comprend alors sa signification entre les mains de Ménage, et ce indépendamment de tout ce qui a pu être dit de la qualité de cette recherche, à une époque où la question de l'"usage", c'est-à-dire de l'altération perpétuelle du visage de la langue, est une question devenue dérangeante pour l'imaginaire linguistique français.

De fait, chez Ménage, on notera que le sentiment de la langue est souvent d'abord celui d'une perpétuation locale, et infiniment précieuse, de certains usages particuliers. Ainsi remarque-t-il dans son épitre liminaire que la langue des paysans se conserve plus longtemps. Souvent, il attire l'attention sur le caractère figé de certains tours ; en regard, sur les formations et déformations 
constantes à l'oeuvre dans la langue. Dans ses Observations de 1672, il ira jusqu'à dire clairement : "Il ne faut point changer certaines façons de parler reçues" 9 . On voit bien alors comment, chez lui, l'habitude d'un repérage de formes anciennes de la langue, et l'habitude, surtout, d'avoir à batailler pour isoler leur physionomie propre, incline à désirer qu'en toutes circonstances l'identité des formes soit préservée. C'est ainsi que la recherche linguistique, pour Ménage, vise à "illustrer" la langue tant par l'investigation historique, que par la constitution du présent en patrimoine. Des "origines", il est passé aux "observations".

Si la sensibilité linguistique de la fin du $\mathrm{XVI}^{\circ}$ siècle et du début du $\mathrm{XVII}^{\circ}$ siècle se proposait volontiers de découvrir dans le passé de la langue la figure de son unité et les principes de sa légitimité, un nouveau courant n'a pas tardé à se faire jour, qui estime préférable de se concentrer sur l'état présent et donc fatalement mouvant de la langue. $\mathrm{C}^{\prime}$ est ainsi que les premières décennies du $\mathrm{XVII}^{\circ}$ siècle furent le théâtre d'un affrontement idéologique où s'opposèrent les deux grandes visions qui, tour à tour dans l'histoire moderne, ont gouverné le système de représentation des langues en Europe : la vision "historiciste", intéressée par la "vie" des langues, leur dynamique, la façon qu'elles ont de se façonner sans cesse à l'intérieur et en dehors d'elles-mêmes (vision dominante au $\mathrm{XVI}^{\circ}$ siècle et au $\mathrm{XIX}^{\circ}$ siècle), et la vision structurale, tournée vers la mise en évidence de systèmes autonomes, immobiles, et possédant une légitimité interne (vision dominante dans la seconde moitié du $\mathrm{XVII}^{\circ}$ siècle, au $\mathrm{XVIII}^{\circ}$ siècle, et au $\mathrm{XX}^{\circ}$ siècle). Ce moment est celui, un

${ }^{9}$ Ménage, Observations sur la langue française, 1672, p. 387. 
peu partout en Europe, particulièrement dans l'Europe méridionale, où le repérage de ce que c'est qu'une langue prend une signification inédite. L'étude de la dissociation de la langue d'autorité qu'est le latin en plusieurs langues modernes comme le français, l'espagnol, l'italien, joue alors un rôle central. Toutefois, on peut dire qu'alors que les recherches de Pasquier et de Ménage s'efforçaient de dégager la cohérence linguistique de l'étude détaillée, patiente et érudite des modifications que le temps avait apportées à l'usage, supposant ainsi qu'une certaine forme de physionomie du français finirait par apparaître en transparence derrière la masse des éléments présents, la nouvelle sensibilité préfère construire l'étude linguistique sur un retranchement net, et parfois vigoureusement affirmé, de l'état présent de la langue hors de son histoire.

F. Brunot a à plusieurs reprises étudié les débuts de cette doctrine nouvelle, et quelques-uns des débats auxquels elle a donné lieu. Il a mis en évidence l'existence, autour de 1618, d'un groupe "puriste" autour de Malherbe et Coeffeteau ${ }^{10}$. A ces noms, il faudrait ajouter celui de Balzac, qui porta le surnom de "restaurateur de la langue française". Enfin, on pourra voir dans les Lettres de Phyllarque à Ariste du Père Goulu (1628), l'un des premiers manifestes de ce courant. Chez tous ces auteurs, la conscience linguistique est motivée par le perfectionnement de l'usage présent de la langue plutôt que par l'observation de la manière dont il évolue. C'est ainsi qu'au travers d'un geste de travail et de culture consciente de la langue (d'où le nom de "puristes"), une forme de synchronie de la langue est de quelque manière arrachée au temps. On notera que, chez tous ces auteurs, revient souvent l'idée d'un

${ }^{10} \mathrm{~F}$. Brunot, La doctrine de Malherbe, P. 582. 
changement radical récent survenu dans la langue (cit Oudin : notre langue est entièrement reformée depuis qq temps). L'étude linguistique peut ainsi tabler sur l'existence d'une nouvelle synchronie à l'oeuvre dans la langue, synchronie qui n'est toutefois pas immédiatement perceptible par l'observateur ordinaire, et qui doit faire l'objet d'un approfondissement par l'étude. Aussi la recherche linguistique menée à l'intérieur de ce nouveau courant est-elle essentiellement tournée vers la grammaire.

Entre 1607 et 1640, une série de nouvelles grammaires du français voient le jour, qui se caractérisent toutes par un souci de rompre avec la tradition latine. (Maupas, Oudin). L"'antiquité" du français n'est plus alors vue comme la source de sa cohérence actuelle : bien au contraire, c'est dans une observation dénuée de toute référence à l'histoire de l'état contemporain de la langue que l'on mise pour comprendre ses principes de fonctionnement. "Autre chose est de composer une Grammaire, \& autre un tresor de la langue"11, déclare notamment Maupas dans l'épitre liminaire de sa Grammaire. Les particularités récentes de l'usage sont enregistrées comme autant de preuves de l'autonomie de sa physionomie. Très nettement, les grammairiens ne sont pas des conservateurs de la langue. C'est ainsi que Maupas se prononce pour le retranchement des lettres inutiles à la prononciation, et qui ne sont que des vestiges de l'étymologie ${ }^{12}$. Il revendique aussi, de façon tant soit peu fanfaronne, le fait de n'avoir pas lu les grammairiens qui ont pu écrire avant lui. Son but est de s'attacher à "une curieuse attention de la naïve

${ }^{11}$ Maupas, Grammaire et syntaxe française, $3^{\circ}$ édition, Rouen, 1632, épitre.

12Maupas, 1607, p. 26. 
propriété de notre parler", "sans m'amuser à esplucher les grammaires", dit-il ; "me semblant plus expédient d'exminer iudicieusement l'usage de nos parties d'oraison, que de recevoir pour reigle les advis d'autruy"13. C'est dire si sa représentation $\mathrm{du}$ français est celle d'une langue toute nouvelle, sans rapport avec les constructions historicistes qui ont pu être élaborées avant lui.

Contre le "passé de la langue", le souci essentiel de ceux qu'on a parfois appelé les "nouveaux grammairiens"14 a clairement été de mettre en place les outils conceptuels destinés à caractériser l'état actuel de la langue. Un des problèmes qui se posent est par exemple celui-ci : comment noter l'usage ? Celui-ci obéit-il à des "règles" ? Le rapport éventuel entre les "règles" et l'histoire fait également l'objet de nombreuses interrogations. Les "règles" actuelles de la langue annulent-elles son historicité ?

A toutes ces questions, on répond souvent par la définition d'un état avéré de la langue en lequel est investie tout l'autorité potentielle que l'on prête au système linguistique. C'est ainsi que, depuis la fin du $\mathrm{XVI}^{\circ}$ siècle, la réflexion linguistique est entraînée dans la recherche d'un état standard de la langue en regard duquel les modifications éventuelles apportées par l'usage puissent apparaitre en variation synchronique, et non comme représentatives d'une avancée ultérieure de la langue dans l'histoire. Pour Pasquier, cet état était représenté par la langue de Ronsard ${ }^{15}$; chez Coeffeteau et Balzac, le critère se fait plus discriminant : pour eux, seul un passé récent est

13Maupas, 1632, épitre.

${ }^{14}$ C'est notamment l'expression de Marie de Gournay. Voir Les Advis, Paris, 1641.

15Pasquier, op. cit., p. 1526. 
encore valable ; enfin pour Vaugelas c'est la langue de Coeffeteau elle-même, assortie à celle de Malherbe, qui représente l'étalon, et marque le moment où, pour ainsi dire, le français sort de l'histoire. Chaque fois, le raisonnement linguistique s'appuie sur la définition d'une microdiachronie, au sein de laquelle le français est vu comme susceptible d'être décrit à la fois dans la cohérence d'un usage et dans un micro espace variationnel (historique) qui n'est pas sans rappeler ceux plus tard définis par W. Labov.

Ce mouvement apparaît comme décisif dans la construction d'une nouvelle idée synchronique de la langue, laquelle ne demande plus ensuite qu'on pose les règles d'un assentiment du locuteur. C'est ainsi, sans doute, qu'il faut interpréter l'aspect tyrannique de l'usage qu'on a souvent remarqué dans les théories de Vaugelas et de ses antécédents immédiats. Si la synchronie de la langue apparaît dans l'homogénéité relevée et abondamment commentée d'un usage avéré, elle se réalise aussi dans la solidarité du locuteur potentiel avec cet usage. Aussi le commentaire grammatical de l'époque enjoint-il aux lecteurs de "parler Malherbe", "parler Balzac", "parler Vaugelas". Il n'en demeure pas moins que les rapports entre autorité et usage restent problématiques. En effet, le "parler Malherbe", le "parler Balzac", le "parler Vaugelas" n'en viennent-ils pas un jour ou l'autre à représenter une force conservatrice contredite par l'usage ? Aussi, dans les Remarques de Vaugelas, trouvera-t-on une discussion constante entre les principes d'autorité et d'usage. Entre ces deux principes Vaugelas, la plupart du temps, et significativement, ne choisit pas. S'il est, dans le fond de sa théorie, essentiellement favorable à l'usage, il comprend néanmoins qu'il n'est plus possible, à l'époque où il écrit, de laisser aller le commentaire linguistique dans le mouvement 
labile du temps. Que les langues dérivent indéfiniment au flux des décennies n'est plus une idée acceptable par ses contemporains.

Toutefois, si autorité il y a, cette autorité sera soigneusement mesurée et circonscrite à quelques figures. Vaugelas, par exemple, prend bien soin de préciser que, si quelques figures choisies comme celles de Malherbe ou de Coeffeteau semblent élues au sein des autres, c'est en raison de leur inscription récente dans le temps, et de ce qu'elles ont pu apporter de spécifique à certaines formes d'usage écrit de la langue (poésie pour Malherbe, prose historique pour Coeffeteau). L'éventuel pouvoir que peuvent prendre les formes écrites de la langue dans la conscience linguistique fait cependant l'objet de restrictions importantes. Lui est opposé, notamment, le contre-pouvoir permanent que constitue l'oral. Ainsi, à l'"achronie" de la langue écrite, comme dit le Bally16, où l'on peut voir additionnés tous les états par lesquels elle a passé, Vaugelas oppose la nécessité de se faire comprendre de la langue parlée, qui la soumet à une synchronie qui est d'abord la synchronie absolue de la communication. De même que Malherbe prétendait ne se référer, pour étalonner sa langue, qu'à celle des "crocheteurs du Port-aux-Foins", Vaugelas, dans la préface de ses Remarques, se lance dans une paradoxale défense de l'oral (mais un oral circonscrit à un espace variationnel particulier, celui de la Cour), à un moment où celui-ci apparaît particulièrement changeant. Cette attitude apparaitra d'autant plus paradoxale que, pour lui, l'écrit doit être un miroir de l'oral. On comprend alors que son système de représentation linguistique est en réalité un savant dosage entre des éléments dynamiques (apportés

${ }^{16}$ Ch. Bally, Le langage et la vie, Genève, Droz, 1942, p. 70. 
par l'usage oral), et des éléments cohésifs (apportés par les "autorités"). Ses Remarques encouragent ainsi une perception synchronique sans nostalgie de la langue, qui se réalise dans une homogénéité pragmatique qui est celle du discours.

Si Vaugelas se réfère à un aussi petit nombre d'autorités, et si ses remarques contiennent aussi peu de notations historiques (pour ainsi dire pas), c'est qu'il n'entend pas figer la langue dans un éternel passé. Dans la référence perpétuelle aux "autorités", en effet, ne convient-il pas de surprendre le geste même qui confère à l'écoulement du temps une force annoblissante, et que la génération des puristes avait précisément dénoncé chez ses prédécesseurs ? Pour Vaugelas, la langue française ne sera pas "illustrée" par l'abondance et l'ancienneté de ses auteurs. Quand il se réfère à quelques grands anciens, il les considère comme des modernes, c'est-à-dire qu'il exploite en eux ce qui peut être utilisé dans une optique contemporaine. L'originalité de cette démarche choquera, sur le moment (elle est l'inverse de la démarche scolastique), et on en comprendra quelques décennies plus tard toutes les conséquences au travers de la célèbre "querelle des anciens et des modernes". Sans doute cette querelle n'aurait-elle pas eu lieu si les auteurs anciens n'avaient pas été sortis par une partie des commentateurs hors de la forme d'éternité où les cantonnait leur ancienneté, et replacés brutalement dans le courant du temps. Dans le domaine de la langue aussi, qui ne remonte pas aussi loin que les champs littéraires et culturels impliqués plus tard, une manière de "querelle" eut lieu, décisive dans l'évolution de la conscience linguistique.

On peut aisément se figurer, en effet, que les positions radicales vis-à-vis de langue qui furent celles des 
grammairiens puristes, et qui trouveront dans les Remarques de Vaugelas leur expression la plus achevée, n'ont pas été sans susciter des réactions. Ces réactions peuvent bien être appréhendées au moyen des différentes vagues de cristallisation polémique qu'engendrèrent, successivement, les déclarations de Malherbe et de Vaugelas. Auteur d'"advis", Marie de Gournay, la nièce de Montaigne défend dans les premières décennies du XVII ${ }^{\circ}$ siècle, et dans une langue qui est encore, mais magnifiquement, la langue forte et imagée du $\mathrm{XVI}^{\circ}$ siècle, l'idée d'une langue perpétuellement vivante, et qui se renouvelle sans cesse d'elle-même17. Elle perçoit les tentatives des "nouveaux grammairiens" comme l'expression d'un désir stérile et inutile d'arrêter le temps, en même temps que comme le fruit d'une esthétique desséchante et normative. Pour elle, les langues sont toujours soumises à l'historicité, mais cette historicité est celle de l'esprit, de la production humaine. Une langue est faite de la somme de qu'ont fait en elle les puissants esprits : "Ce langage consiste encore de tout ce qu'une prudente, vigoureuse \& superbe audace, peut édifier sur cet amas, informant $\&$ paistrissant à toutes mains cette masse" 18 . Une langue, c'est aussi un fond, "le hors de page d'une langue", dit-elle, "depuis lequel, comme nous disions d'arrivée, elle peut avancer, non reculer ny vieillir"19. En d'autres phrases saisissantes, elle évoque la manière de marche forcée des langues, qui les entraîne toujours plus vers l'avenir : "la presente nous desgoute de la precedente" 20 , dit-elle. C'est

${ }^{17}$ Marie de Gournay, Les Advis, Paris, 1641, p. 79-80.

18Ibid., p. 453.

19Ibid.

${ }^{20}$ Ibid. 
cette obsolescence qui précisément empêche la langue de "vieillir". S'opposant à la minutie grammaticale et au souci de figer une certaine physionomie de la langue, elle défend au contraire son actualité constante, sa faculté permanente de s'amender elle-même.

Une partie de ces arguments sera réutilisée au moment de la parution des Remarques de Vaugelas, lesquelles seront perçues par beaucoup comme l'expression d'une négation de l'histoire de la langue au profit d'une valorisation à courte vue d'un usage mondain et sans intérêt. "On est toujours enfant dans sa langue quand on ne lit que les auteurs de son temps et que l'on ne parle que la langue de sa nourrice", dira Ménage, par exemple 21 , soulignant le caractère limité, voire infantile, du schéma synchronique mis en avant par Vaugelas. Scipion Dupleix, dans un ouvrage intitulé significativement Liberté de la langue française dans sa pureté, s'élèvera contre la tyrannie de l'usage 22 . Il développe l'idée d'une sorte de hasard, régissant de façon totalement arbitraire de ce qui "reste" (nous soulignons) dans une langue, et de ce qui ne reste pas $^{23}$. Il note également le paradoxe qui consiste, pour Vaugelas, à recourir au terme de "vieilli", ("partant"), contradictoire avec sa définition de l'usage.

Dans ses Nouvelles remarques sur la LF, lettres écrites à Naudé à propos de Vaugelas, de même, La Mothe le Vayer, s'oppose à la manière qu'a Vaugelas de juger des expressions de langue en les retirant de leurs situations de discours. Il remarque que la langue d'Homère mêlait tous

${ }^{21}$ Menagiana, II, 291.

22Scipion Dupleix, Liberté de la langue française dans sa pureté, 1650, p. 9.

${ }^{23}$ Ibid., p. 62. 
les styles et tous les dialectes, et recourait parfois à des archaïsmes qui l'étaient déjà à l'époque. Ainsi, il défend l'idée d'une hétérogénéité foncière de l'écriture. Le principal reproche qu'il adresse à Vaugelas est de retirer la langue hors du temps. De cette manière, aucune nuance n'est plus appréciable dans sa signification réelle. Aussi bien Vaugelas ne peut-il trancher -et c'est là un grave défaut pour La Mothe Le Vayer- qu'au moyen d'une discussion entre autorité et usage qui exclut de plus tout recours à la "raison".

Sans aucun doute, l'oeuvre de Vaugelas constitue un tournant dans la perception linguistique du $\mathrm{XVII}^{\circ}$ siècle. Il nous est difficile de mesurer aujourd'hui à quel point les positions qu'elle défend pouvaient paraitre à l'époque problématiques et difficiles à soutenir. Beaucoup de commentateurs se sont montrés désorientés par l'espèce de "flottement libre" dans lequel Vaugelas laissait la langue, sans référence, ni à son histoire, ni à la "raison". Il est clair que Vaugelas entendait représenter dans l'état synchronique d'une langue une force intrinsèque capable de démentir les théories et les décisions. Paradoxalement, c'est dans le sens d'un durcissement que sa position fut interprétée. En effet, pour beaucoup, sa résignation confiante face au mouvement de l'histoire et à l'obsolescence régulière de la langue pouvait passer pour un renoncement à tout contrôle. C'est ainsi que nombre de ses hypothèses qui, quand on en lit bien le texte, témoignent d'une sensibilité à la langue qui n'est pas si foncièrement éloignée que cela de celle de Marie de Gournay, furent revues dans un sens plus normatif qui devait opposer au passage du temps une certaine forme de résistance. 
Ainsi, dans les Entretiens d'Ariste et d'Eugène (1662), Bouhours se propose-t-il d'effectuer une synthèse entre la perspective historique de Pasquier, et la description synchronique initiée par Vaugelas. Recopiant de grands passages des Recherches de la France ${ }^{24}$, il les insère dans un dispositif qui semble conférer aux données historiques un rôle de confirmation rétrospective des hypothèses formulées sur l'état actuel de la langue française. C'est ainsi qu'un motif ingénieux vient aider à concilier l'idée d'un mouvement des langues dans le temps et la présupposition d'une force capable de les aider à y résister : le "génie" des langues. Pour Bouhours, tout a changé dans l'histoire de la langue, sauf la "naïveté", qu'on retrouve en ancien français. Il reprend à son compte l'idée d'une "naïve françoeze"25 qui serait une sorte de langue française archétypale naturelle aux Français. Ce "génie", cette "naïveté", ne sont ainsi pas incompatibles avec l'histoire. Non seulement le mouvement historique n'altère pas le génie, mais il semble même le rendre de plus en plus visible.

A vrai dire, la force conférée au "génie" sera de plus en plus considérée, au fur et à mesure que l'on avance dans le siècle, comme l'antidote idéal face aux pouvoirs de 1"'usage", lequel est perçu comme une force destructrice à un moment où l'on commence à estimer sérieusement que la langue française a atteint un point de perfection. En 1675, un projet d'arc de triomphe va cristalliser autour de lui toutes les interrogations qui traversent la conscience linguistique quant au statut des langues face à l'histoire. Pour la première fois, on envisage que l'inscription gravée

${ }^{24}$ Bouhours, Entretiens, Paris, 1962, p. 108 et suivantes.

${ }^{25}$ Concept qui remonte sans doute à Meigret ; voir $\mathrm{D}$. Trudeau, op. cit., p. 71. Voir également Jean Godard, La Langue françoise, Lyon, Jullieron, 1620. 
dans le marbre le soit en français, et non plus en latin. Cette petite "révolution" signale bien évidemment qu'une importance politique nouvelle est donnée au français. Elle est l'occasion, par ailleurs, d'une querelle entre défenseurs du latin et défenseurs du français qui permet bien de se représenter comment les problématiques linguistiques (langues vivantes/langues mortes) sont articulées face au temps. Le Père Lucas et Bourzeys défendirent que les inscriptions devaient être faites en latin. Dans un discours académique prononcé le 23 décembre 1676, Tallemant le Jeune s'y opposa. De son côté, dans deux ouvrages consécutifs, l'un court et polémique (Deffense de la langue françoise pour l'inscription de l'arc de triomphe, 1676), l'autre long et argumenté (De l'Excellence de la langue françoise, 1683), Charpentier s'est montré celui qui a pris l'affaire le plus au sérieux.

Son idée est simple, elle est, ainsi que le formule le titre du chapitre XVII, "Que la langue française est présentement immortelle" 26 . Il relève, dans le commentaire qui a été fait jusqu'à présent des langues vernaculaires, "une honteuse dépendance de l'usage"27. Par là, il s'inscrit dans la lignée des Réflexions sur la tyrannie de l'usage contemporaines d'Arnauld (AV). Rappelons que l'usage était souvent qualifié, depuis Vaugelas, de "tyran des langues". Contre cette perception de la langue, Charpentier suppose communément partagé un désir d'immortalité. Il note qu'au $\mathrm{XVII}^{\circ}$ siècle, ce désir s'est essentiellement manifesté dans l'accès tacite, progressivement entériné, du latin au statut de langue immortelle. Charpentier entend ici

${ }^{26}$ Charpentier, De l'excellence de la langue françoise, 1683, p. 346.

${ }^{27}$ Ibid. 
dissocier le concept d"'immortalité de la langue", qui est clairement issu du statut particulier du latin au XVII siècle, mais auquel Charpentier voudrait donner une valeur générale, du fait que la langue soit à proprement parler morte ou vivante. "Il est donc indifférent à une langue pour être immortelle, qu'elle vive ou qu'elle ne vive plus, pourvu qu'elle soit écrite dans des ouvrages qui puissent subsister"28, dit-il. Il intitule par ailleurs son chapitre XVIII : "Que la langue française est présentement fixe au même sens que l'est la Romaine"29.

Ainsi Charpentier s'oppose-t-il à l'usage perpétuellement reconduit du latin dans les inscriptions sur la base que sa physionomie serait sortie du temps, et ne serait plus susceptible de recevoir de modification. Pour lui, si le français est encore en usage, cela ne signifie pas que l'usage doive continuer à altérer sa physionomie et la soumettre à la menace de l'historicité. L'activité métalinguistique, entre autres, a pour but de circonscrire l'usage et d'en donner une description qui s'appuie sur d'autres principes qu'un simple enregistrement des "mots à la mode", selon l'expression de F. de Callières ${ }^{30}$. Le travail académique du dictionnaire, par exemple, est clairement vu par Charpentier comme une forme de résistance au temps, visant à donner "une image incorruptible de la beauté de notre langue" 31 . Chez les défenseurs du latin, il relève l'aberration selon laquelle il faudrait attendre, pour que les langues vivantes soient propres à être employées pour des

28Ibid, p. 357.

${ }^{29}$ Ibid., p. 360.

${ }^{30} \mathrm{~F}$. de Callières, Des mots à la mode, La Haye, A. Troyel, 1692.

${ }^{31}$ Charpentier, Deffense de la langue françoise, p. 323. 
inscriptions, qu'elles soient des langues mortes ${ }^{32}$. Dans l'état actuel du français, il considère que se trouve enfermé un usage vivant de la langue capable de faire néanmoins sortir celle-ci de l'historicité.

En très peu d'années, entre les Entretiens de Bouhours et la querelle des inscriptions, la sensibilité à l'égard du statut historique de la langue française a donc considérablement changé. Entre temps, Le Laboureur a publié ses succincts et péremptoires Avantages de la langue française sur la langue latine (1667), dans lesquels il lutte avec vigueur contre un reste de prévention en faveur du latin. Pour Le Laboureur, la langue française présente désormais un état achevé. "Je ne pense pas qu'elle change guère davantage" 33 , dit-il. Les années 70 sont l'occasion d'un déferlement de triomphalisme auquel le genre du discours académique donne un moyen d'expression privilégié. Une scission s'effectue, dans la représentation linguistique, entre l'histoire de la langue, et un état qu'on pourrait dire an-historique de cette langue, qui en contient sous forme concentrée le génie, et ne donne pas de prise à l'usage. [Notre langue], dit l'abbé de Montigny dans son discours de 1670, "n'est plus cette gauloise, \& cette gothique" 34 . L'histoire de la langue est donc vue comme un état antérieur de cette langue : c'est cette rupture radicale qui est susceptible de garantir la sortie du temps de l'usage actuel.

Celui-ci, par les soins de l'entreprise académique, est vu comme étant désormais établi. "Or comment peut-on

${ }^{32}$ Charpentier, De l'excellence de la langue françoise, p. 354.

${ }^{33}$ Le Laboureur, Avantages, p. 10.

${ }^{34}$ Recueil des harangues prononcées par Messieurs de l'Académie, 1709, p. 149. 
confier des actions immortelles à des langues toujours incertaines et toujours changeantes ; \& la nôtre en particulier pouvait-elle promettre l'immortalité, elle dont nous voions tous les jours passer les beautés, et qui devenait barbare à la France même dans le cours de peu d'années ?" , lit-on dans le discours de l'évêque de Condom, 167135. "La langue française par vos soins est parvenue à sa dernière perfection : c'est à vous maintenant de pratiquer le bon usage que vous avez établi. Considérez le juste rapport qu'il $\mathrm{y}$ a entre les trois siècles académiques dont je vous ai parlé, et ajoutez-y que les langues grecque et latine avaient aussi dans ce même temps atteint leur dernière pureté.", lit-on dans celui de Tallemant le Jeune en $1675^{36}$. "Qu'on ne parle plus de changement dans notre langue, elle est fixée à jamais ...", dit le même Tallemant dans son discours de $1676^{37}$.

Sans nul doute, par conséquent, les commentateurs de cette fin du $\mathrm{XVII}^{\circ}$ siècle se sont montrés convaincus qu'il était possible, avec quelque intervention, de modifier à ce point les contours de la langue que celle-ci en acquière une physionomie intemporelle, à l'instar des monuments de pierre et de marbre au moyen desquels on se représentait volontiers que les anciennes civilisations avaient résisté au temps. A ce titre, la querelle des inscriptions se révèle particulièrement significative. La langue est revêtue d'un caractère monumental qui est censé en décupler la force signifiante. Toutefois, nombre de commentateurs notent que cette "monumentalisation" ne peut s'effectuer dans n'importe quelles conditions : il convient que la langue elle-

\author{
35Ibid., p. 219. \\ 36Ibid.,p. 376. \\ 37Ibid., 422.
}


même possède les caractères nécessaires à sa survie. Inscrire dans le marbre une langue sujette encore au changement revient à détruire l'effet monumental. Pour l'abbé Galloys, qui, dans son discours à la réception de Dangeau en1682, revient encore, sur la question des inscriptions, ce fut le tort des Egyptiens..., de n'avoir pas assuré la survie de leur langue en dépit de leurs talents constructeurs 38 .

Dans les années 80 , on peut donc dire que, l'affaire de l'arc de triomphe ayant cristallisé autour d'elle des sentiments épars dans l'air du temps, l'enjeu décisif qui gouverne les problématiques grammaticales dans la conscience linguistique nationale est un enjeu de mémoire et de résistance face au temps.

Entre le relativisme historique du début du siècle et l'affirmation triomphaliste d'uns "sortie de l'histoire" du français à la fin du siècle, il semble donc que le chemin ait été à la fois long et rapidement parcouru. Dans l'histoire des représentations de notre langue, la fin du $\mathrm{XVII}^{\circ}$ siècle est souvent citée comme l'un des moments où l'idée d'une "langue " transcendante à sa réalisation discursive a eu le plus de force. Indiscutablement, ce moment prend une force nouvelle si on l'examine à la lumière de ce qu'aura produit une autre grande époque d' "an-historicisme" : le $\mathrm{XX}^{\circ}$ siècle structuraliste, que l'on pourra comprendre dans une forme étendue où l'on verrait se rejoindre les abstractions de la langue tirées des théories de Saussure et Guillaume, et l'intemporalité du raisonnement linguistique supposée par la grammaire générative de Chomsky. Au XVII ${ }^{\circ}$ siècle, la

38Ibid., p. 511. 
sortie hors du temps du regard porté sur la langue pourra se voir illustrée de façon significative par les concepts utilisés en grammaire : aux traditionnelles "causes" néo-latines invoquées par Scaliger vont succéder les "principes" exploités au $\mathrm{XVIII}^{\circ}$ siècle. Dans la langue, on pourra prendre l'habitude de considérer une production humaine régie par des lois intemporelles, et dont l'autonomie, singulièrement dérangeante pour peu qu'on la saisisse dans ses capacités aliénantes, est considérée comme une protection contre le temps. On comprend alors la fascination que le commentaire linguistique a pu exercer au XVII ${ }^{\circ}$ siècle : commenter la langue, c'était commenter les chances qu'avait une civilisation d'assurer sa survie.

A examiner de plus près les débats que cette évolution aura suscitée, toutefois, on ne pourra qu'être frappé par la consistance de l'opposition en faveur de l'histoire. Cette opposition, il faut le dire, est de moins en moins visible au fur et à mesure qu'on avance dans le siècle. Sans doute a-t-elle été " absorbée", pour ainsi dire, par la dynamique du mouvement central qui s'organisait autour des représentations du français, piégée, en particulier, par l'habileté synthétique d'un Bouhours et sa capacité à réconcilier entre eux les suggestions de l'inconscient collectif et le patrimoine de la réflexion. Au final, on voit bien comment, dans notre pays, le concept de "langue" est un artefact qui fut arraché -autant qu'à l'espace (aux frontières) -au temps (à l'histoire). Une partie de l'histoire de la langue était vieillie, une partie restait toujours actuelle ; une partie se voyait transformée en scories, laissées sur le bord de la route sans espoir qu'on y revienne jamais, une partie portait en elle la préparation du "génie " qui allait assurer à la langue sa pérennité. Cette opération de " tri ", à laquelle se livrèrent avec beaucoup de 
passion la plupart des commentateurs du $\mathrm{XVII}^{\circ}$, révèle à quel point la description linguistique est souvent animée par un conflit interne entre structures incompatibles. La complémentarité rêvée entre synchronie et diachronie, sur laquelle s'est appuyée une bonne partie du commentaire de notre siècle sur le français n'est-elle pas le signe secret que la diachronie, alors, était conçue comme désormais inopérante, "finie", associée à l' "éternel passé de la langue "?

Gilles Siouffi

Université Paul Valéry, Montpellier III

\section{Bibliographie :}

\section{1/ Sources :}

-BARY, René, La Rhétorique françoise, où pour principale augmentation l'on trouve les secrets de notre langue, Paris Le Petit, 1665.

-BOUHOURS, Dominique, Les Entretiens d'Ariste et d'Eugène, Paris, éditions de Cluny, 1962.

-CHARPENTIER, François, Deffense de la langue françoise pour l'inscription de l'Arc de Triomphe, Paris, $\mathrm{Cl}$. Barbin, 1676. 
-CHARPENTIER, François, De l'Excellence de la langue française, Paris, Vve Bilaine, 1683.

-Commentaires sur les Remarques de Vaugelas, éd. J. Streicher, 2 volumes, Genève, Slatkine, 1970.

-DUPLEIX, Scipion, Liberté de la langue françoise dans sa pureté, Paris, Denys Bechet, 1651.

-GOURNAY, Marie de, Les Advis, ou les Présens de la Demoiselle de Gournay, $3^{\circ}$ éd, Paris, Jean du Bray, 1641.

-LE LABOUREUR, Louis, Les Avantages de la langue française sur la langue latine, Paris, F. Lambert, 1667.

-MÉNAGE, Gilles, Les Origines de la langue françoise, Paris, Courbé, 1650.

-MÉNAGE, Gilles, Observations de M. Ménage sur la langue française, Paris, C. Barbin, 1672.

-NICOT, Jean, Thresor de la langue françoyse, tant ancienne que moderne, Paris, 1606, réimpression fac-similé Paris, Le Temps, 1979.

-PASQUIER, Etienne, Les Recherches de la France, édition critique dirigée par M-M. Fragonard et F. Roudaut, 3 volumes, Paris, Champion, 1996.

-Recueil des harangues prononcées par Messieurs de l'Académie françoise dans leurs réceptions, \& en d'autres occasions différentes, depuis l'establissement de l'Académie jusqu'à present, Amsterdam, Aux dépens de la Compagnie, 1709, 2 vol.

-VAUGELAS, Claude Favre de, Remarques sur la langue française, édition J. Streicher, Genève, Droz, 1936.

2/ Critique :

-BRUNOT, Ferdinand, La doctrine de Malherbe d'après son commentaire sur Desportes, Paris, A. Colin, 1969. 
-DEMAIZIERE, Colette, "La situation linguistique et le plaidoyer pour la langue française d'Estienne Pasquier", in Etienne Pasquier et ses Recherches de la France, Paris, Presses de l'ENS, 1991, 23-33.

-DROIXHE, Daniel, La linguistique et l'appel à l'histoire, Genève, Droz, 1978.

-TRUDEAU, Danielle, Les inventeurs du bon usage, Paris, Minuit, 1992. 